\title{
A Study on the Complexity of a New Chaotic Financial System
}

\author{
Yi Liao $\mathbb{D},{ }^{1}$ Yiran Zhou $\left(\mathbb{D},{ }^{1}\right.$ Fei Xu $\mathbb{D},{ }^{2}$ and Xiao-Bao Shu ${ }^{3}$ \\ ${ }^{1}$ School of Business Administration, Southwestern University of Finance and Economics, Chengdu, Sichuan 610074, China \\ ${ }^{2}$ Department of Mathematics, Wilfrid Laurier University, Waterloo, Ontario, Canada \\ ${ }^{3}$ Department of Mathematics, Hunan University, Changsha 410082, China
}

Correspondence should be addressed to Fei Xu; fxu.feixu@gmail.com

Received 17 August 2020; Revised 19 September 2020; Accepted 25 September 2020; Published 10 October 2020

Academic Editor: Yongjian Liu

Copyright (C) 2020 Yi Liao et al. This is an open access article distributed under the Creative Commons Attribution License, which permits unrestricted use, distribution, and reproduction in any medium, provided the original work is properly cited.

\begin{abstract}
The interaction of elements in a financial system can exhibit complex dynamic behaviours. In this article, we use a system of differential equations to model the evolution of a financial system and study its complexity. Numerical simulations show that the system exhibits a variety of rich dynamic behaviours, including chaos. Bifurcation diagrams show that the system behaves chaotically over a wide range of system parameters.
\end{abstract}

\section{Introduction}

Chaotic attractors have attracted the attention of scholars in different fields. Chaos has a wide range of applications in natural science and engineering. The first chaotic system is the Lorenz system [1], which was introduced for weather forecast. Later investigations found that there exist chaotic attractors in the chemical reaction model [2] and electrical circuit [3]. Investigation shows that the generalized Lorenz systems family includes the classical Lorenz system [1], the Chen system [4], and the Lü system [5]. Zhang et al. studied the Lü system and showed that the Lü system is globally bounded using dynamical systems theory [6]. In addition, chaos has a wide range of applications in cryptography and engineering $[4,5,7]$. Investigations have shown that a variety of ecological systems display chaotic behaviours $[8,9]$. In recent years, chaotic systems that display multiscroll [10-12] and multiwing [13] chaotic attractors were considered in the literature. Zhang et al. studied the dynamics of a New 5D Hyperchaotic system of Lorenz type and obtained the global exponential attractive set and the ultimate bound set for this system of Lorenz Type [14]. Such chaotic systems display more complex dynamical behaviours and have potential applications in engineering.

A variety of economic and financial systems display complex behaviours. Benhabib et al. studied a series of market models and showed that such systems behave chaotically [15-17]. Grandmont [18] studied the chaos in endogenous competitive business cycles and performed bifurcation analysis. Brock analysed the maximum Lyapunov exponent of quarterly US real GNP data (1947-1985) and confirmed the chaotic behaviours in the data [19].

Research shows that there is chaos in market models, financial systems, and supply chains. For example, Huang and $\mathrm{Li}$ used system of differential equations to model a financial system, in which the interactions between interest rate, investment demand, and price index are considered [20]. This model was further investigated by $\mathrm{Ma}$ and Chen $[21,22]$. The authors performed a complete study on the dynamical properties of the model. $\mathrm{Xu}$, et al. studied the interaction effect among several financial factors in a financial system using mathematical models [23]. Their investigation showed that such model displays rich dynamical behaviours including chaos. Synchronization of the model were considered in their work. Zhang et al. studied a 4D Chaos Financial System [24]. Investigations show that supply chain systems also display a variety of complex dynamical behaviours [25-27].

In this work, we construct a mathematical model to study a financial system using differential equations. The model captures the interaction between a variety of financial factors. The rest of this paper is organized as follows. In 
Section 2, we formulate the model. In Section 3, we perform numerical simulations to study the complexity of the model. Conclusions are drawn in Section 4.

\section{Model Formulation}

In this section, we consider the model formulation of the financial system. Huang and Li proposed a model using differential equations to investigate the behaviour of a financial system containing interest rate, investment demand, and price index [20]. In the following, based on the model presented in [20], we present an alternative model to consider the interplay between the interest rate $x(t)$, the investment demand $y(t)$, and the price index $z(t)$. The model is given by

$$
\begin{aligned}
& \frac{\mathrm{d} x}{\mathrm{~d} t}=g z+(y-a) x, \\
& \frac{\mathrm{d} y}{\mathrm{~d} t}=-b y^{2}-s x^{2}+r, \\
& \frac{\mathrm{d} z}{\mathrm{~d} t}=-c z-\beta x-p y,
\end{aligned}
$$

where $a, b, c, p, r, s$, and $\beta$ are constants. System (1) assumes that the rate of change of interest rate is proportional to the price index. Investment demand significantly influences the interest rate. On one hand, if the investment demand exceeds the saving amount, the bank will increase the interest rate. On the other hand, when the investment demand is low, the saving amount may exceed the investment demand. In this case, the bank has to reduce the interest rate to encourage loan. For the investment demand $y(t)$, it admits a natural growth rate of $r$. If the interest rate is increased, the investment will be negatively affected. That is to say, the growth rate of the investment demand will decrease. Excessive investment demand will lead to the decrease of the change rate of the investment demand. Therefore, we have $-b y^{2}$ term, where we assume that the rate of change of investment demand is negatively correlated with the square of investment demand. It is assumed that the interest rate has negative correlation on the change rate of the price index with rate $\beta$. The change rate of price index will decrease with the increase of investment demand. Compared with the chaotic system proposed in [21], the price index $z(t)$ in the system proposed in this paper is affected by the investment demand $y(t)$. On one hand, an increase in investment demand will inevitably lead to an increase in production, which will lead to a decrease in price. On the other hand, a decrease in investment demand $y(t)$ will lead to a decrease in the amount of products in the market, leading to an increase in the average price.

\section{Dynamical Behaviour of the Model}

In this section, we investigate the dynamical behaviours of the model (1). System (1) displays chaotic behaviours under the following parameter combinations: $a=0.3, b=0.02$, $c=1, r=1, s=0.1, p=0.05, g=1.2$, and $\beta=1$. With these parameters, the system admits the following equilibrium points:

$$
\begin{aligned}
& E_{1}=(0.04949849664,-7.070201517,0.3040115792), \\
& E_{2}=(0.07616084207,7.069016737,-0.4296116789), \\
& E_{3}=(3.087391472,1.529728564,-3.163877901), \\
& E_{4}=(-3.093050811,1.471456216,3.019478000) .
\end{aligned}
$$

Linearizing system (1) at any of its equilibrium $E_{*}=\left(x^{*}, y^{*}, z^{*}\right)$ yields

$$
J=\left[\begin{array}{ccc}
y^{*}-a & x^{*} & g \\
-2 s x^{*} & -2 b y^{*} & 0 \\
-\beta & -p & -c
\end{array}\right] .
$$

By calculating the corresponding eigenvalues of $E_{1}, E_{2}$, $E_{3}$, and $E_{4}$, we find that these equilibrium points are all unstable.

We then study system (1) numerically to investigate its dynamic behaviours. The phase portrait of model (1) is shown in Figure 1, which shows that the trajectory of the system is a chaotic attractor. Since the model describes the interaction among the three financial factors, the complicated behaviours of the model imply that the interplay between these financial factors causes complex behaviours. It is well known that one of the characteristics of a chaotic system is its pseudo-random behaviour. Next, we examine the time history of the system. As is shown in Figure 2, the time history of system (1) is unpredictable and displays complex and pseudorandom behaviours.

Another characteristic of chaotic systems is their sensitivity to initial conditions. That is to say, for the same system, for the same parameters, different initial values will lead to completely different system behaviours. Here, we use numerical method to show the sensitivity to initial values of the financial system (1). As shown in Figure 3, for system (1), we use initial values $(1.2,1.5,1.6)$ to obtain the blue trajectory. Under the same parameters, system (1) with initial values $(0.2,0.5,0.6)$ yields red trajectory. From Figure 3 , we can see that the trajectories corresponding to different initial conditions are completely different, i.e., chaotic system (1) is sensitive to initial conditions.

Model (1) displays a variety of complicated dynamical behaviours. In the following, we create bifurcation diagrams to illustrate that the system behaves chaotically over a wide range of parameters. In the bifurcation diagram, we show the peak values of state variable $y$ with the variation of $r, g$, and $\beta$.

The bifurcation diagram of system (1) with the variation of $r$ is shown in Figure 4. We find that the system behaves chaotically for $r \in[0.6,1.8]$. In this model, $r$ is the natural growth rate of the investment demand $y(t)$. The simulation results show that increasing $r$ can effectively reduce the complicate behaviours of the model. Parameter $g$ significantly influences the behaviour of system (1) as well. Bifurcation diagram Figure 5 implies that the system behaves chaotically for $g \in[0.85,1.5]$. Next, we consider the dynamic 


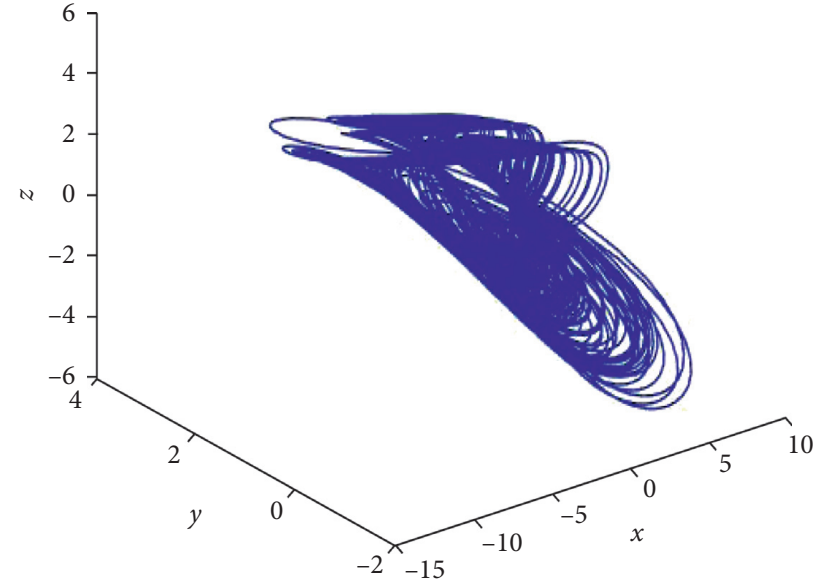

(a)

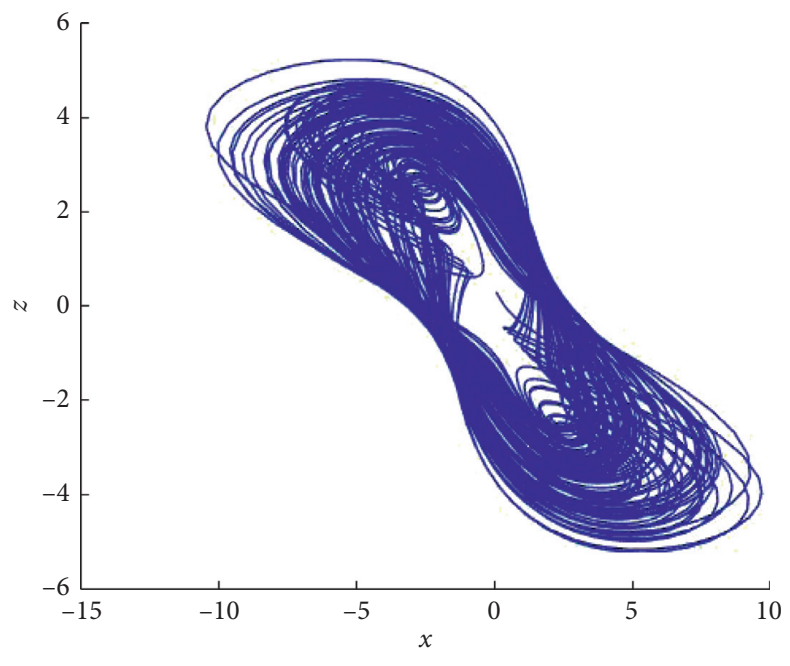

(c)

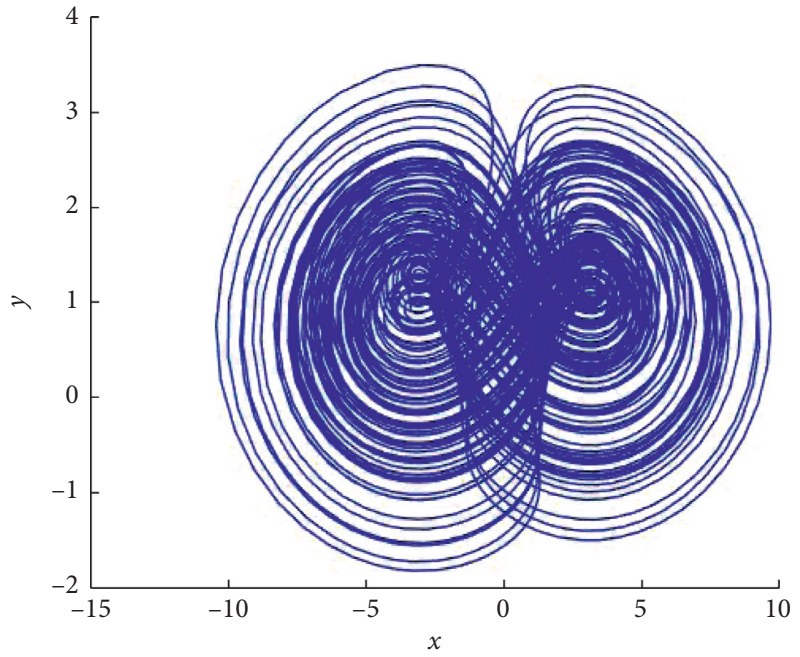

(b)

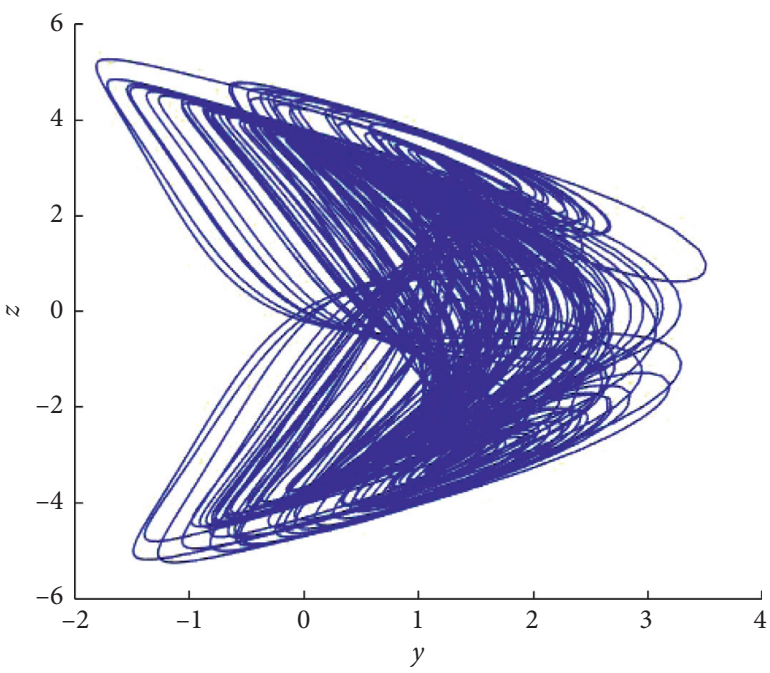

(d)

FIGURE 1: Simulation results of system (1) when $a=0.3, b=0.02, c=1, r=1, s=0.1, p=0.05, g=1.2$, and $\beta=1$. (a) In the $x$ - $y$ - $z$ space, (b) projected on the $x-y$ plane, (c) projected on the $x-z$ plane, and (d) projected on the $y$ - $z$ plane.

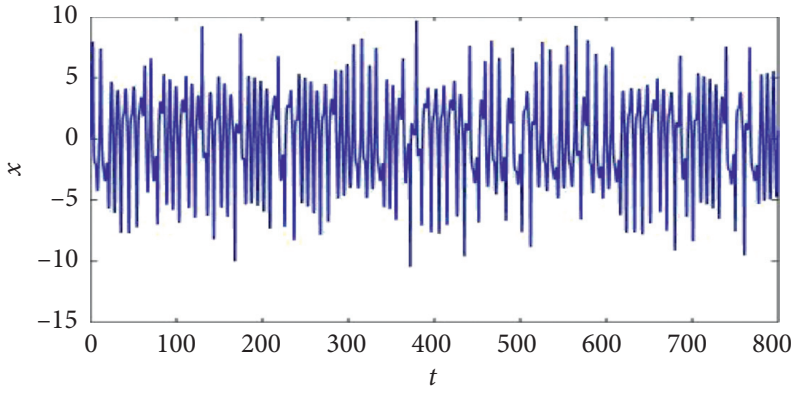

(a)

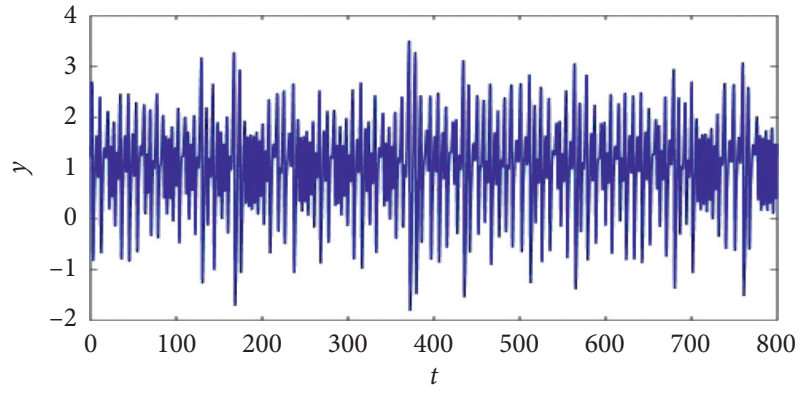

(b)

Figure 2: Continued. 


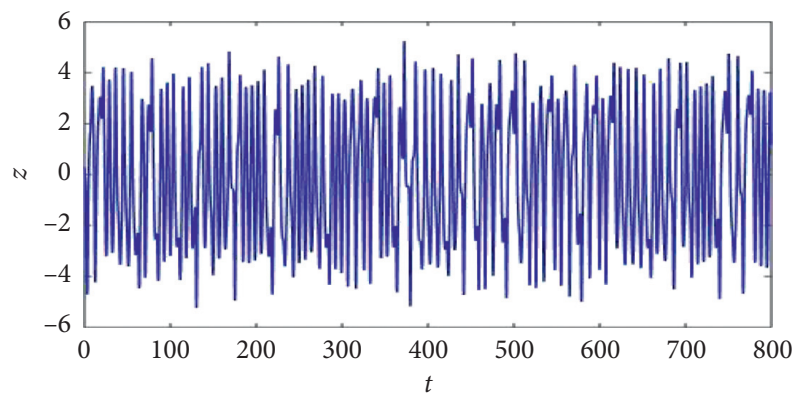

(c)

Figure 2: Time history of system (1) for $a=0.3, b=0.02, c=1, r=1, s=0.1, p=0.05, g=1.2$, and $\beta=1$. (a) Time history of $x$, (b) time history of $y$, and (c) time history of $z$.

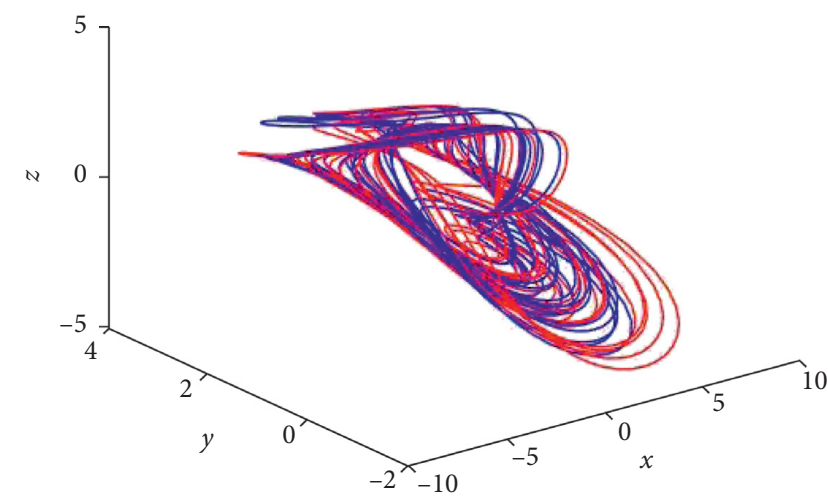

FIGURE 3: Simulation results of system (1) for $a=0.3, b=0.02, c=1$, $r=1, s=0.1, p=0.05, g=1.2$, and $\beta=1$ in the $x-y-z$ space, where the blue trajectory has the initial condition $(1.2,1.5,1.6)$ and the red trajectory has the initial condition $(0.2,0.5,0.6)$.

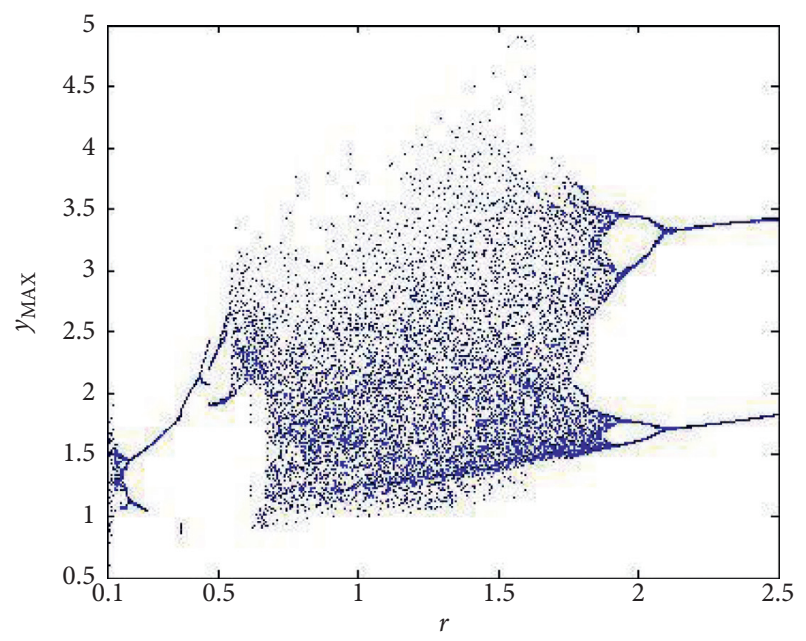

FIgURE 4: Bifurcation diagram of system (1) as $r$ varies from 0.1 to 2.5. The values of all the other parameters are the same as those in Figure 1.

behaviours of system (1) with the variation of $\beta$. As shown in Figure 6 , the system displays chaotic attractors for $\beta \in[0.72$, $1.26]$.

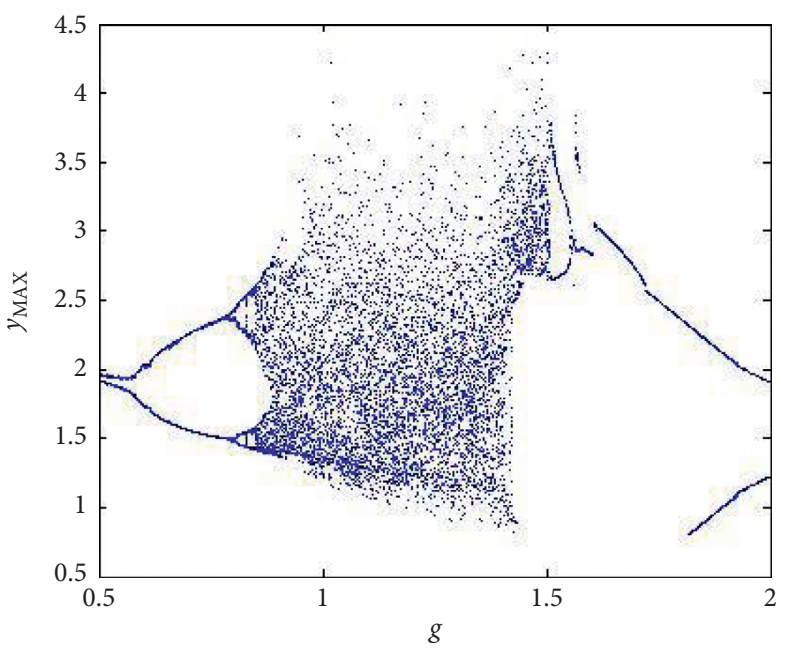

FIGURE 5: Bifurcation diagram of system (1) as $g$ varies from 0.5 to 2. The values of all the other parameters are the same as those in Figure 1.

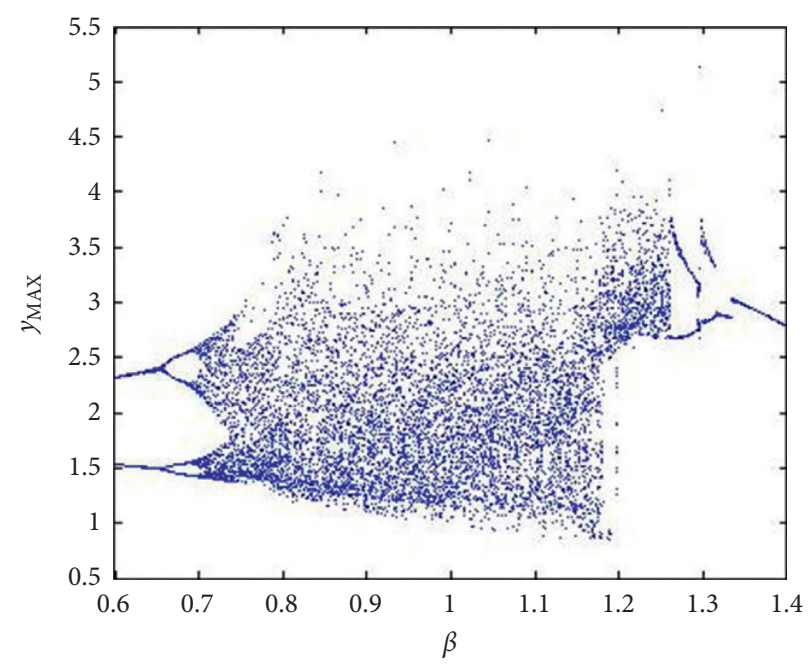

Figure 6: Bifurcation diagram of system (1) as $\beta$ varies from 0.6 to 1.4. The values of all the other parameters are the same as those in Figure 1. 


\section{Conclusions}

In this paper, we propose a system of differential equations to model the interactions between factors in a financial system. We find that the behaviours of the system are unpredictable and sensitive to the initial conditions, and the time history of the system displays pseudo-random behaviours, which implies the system has chaotic behaviours. The financial system displays a variety of complicated dynamical behaviours including chaos and period-doubling bifurcations. Bifurcation diagrams are created to show that the system behaves chaotically over a wide range of system parameters, which indicates that the interactions among the three factors in the financial model cause complex behaviours.

\section{Data Availability}

All the data are included in the article.

\section{Conflicts of Interest}

The authors declare that there are no conflicts of interest regarding the publication of this paper.

\section{Acknowledgments}

This work was supported by the Innovation Platforms Open Foundation of Hunan Educational Committee (grant no. 541109100002).

\section{References}

[1] E. N. Lorenz, "Deterministic nonperiodic flow," Journal of the Atmospheric Sciences, vol. 20, no. 2, pp. 130-141, 1963.

[2] O. E. Rössler, "An equation for continuous chaos," Physics Letters A, vol. 57, no. 5, pp. 397-398, 1976.

[3] L. Chua, M. Komuro, and T. Matsumoto, "The double scroll family," IEEE Transactions on Circuits and Systems, vol. 33, no. 11, pp. 1072-1118, 1986.

[4] G. Chen and T. Ueta, "Yet another chaotic attractor," International Journal of Bifurcation and Chaos, vol. 9, no. 7, pp. 1465-1466, 1999.

[5] J. Lü and G. Chen, "A new chaotic attractor coined," International Journal of Bifurcation and Chaos, vol. 12, no. 3, pp. 659-661, 2002.

[6] F. Zhang, X. Liao, and G. Zhang, "On the global boundedness of the Lü system," Applied Mathematics and Computation, vol. 284, pp. 332-339, 2016.

[7] X. Wang, L. Teng, and X. Qin, "A novel colour image encryption algorithm based on chaos," Signal Processing, vol. 92, no. 4, pp. 1101-1108, 2012.

[8] A. Hastings and T. Powell, "Chaos in a three-species food chain," Ecology, vol. 72, no. 3, pp. 896-903, 1991.

[9] F. Xu, R. Cressman, R. Cressman, and V. Křivan, "Evolution of mobility in predator-prey systems," Discrete \& Continuous Dynamical Systems - B, vol. 19, no. 10, pp. 3397-3432, 2014.

[10] F. Xu, P. Yu, and X. Liao, "Global analysis on n-scroll chaotic attractors of modified Chua's circuit," International Journal of Bifurcation and Chaos, vol. 19, no. 1, pp. 135-157, 2009.

[11] Y. Ma, Y. Li, G. Zhao, P. Zeng, and Y. Yang, "Research on grid scroll chaotic extended sequence algorithm and its circuit implementation for F-OFDM System," Complexity, vol. 2019, Article ID 5904801, 7 pages, 2019.

[12] X. Hu and P. Zhou, "Coexisting three-scroll and four-scroll chaotic attractors in a fractional-order system by a three-scroll integer-order memristive chaotic system and chaos control," Complexity, vol. 2020, Article ID 5796529, 7 pages, 2020.

[13] F. Xu, "A class of integer order and fractional order hyperchaotic systems via the Chen system," International Journal of Bifurcation and Chaos, vol. 26, no. 6, 2016.

[14] F. C. Zhang, R. Chen, X. Y. Wang, X. S. Chen, C. L. Mu, and X. F. Liao, "Dynamics of a new 5D hyperchaotic system of Lorenz type," International Journal of Bifurcation and Chaos, vol. 28, no. 3, 2018.

[15] J. Benhabib and K. Nishimura, "The hopf bifurcation and the existence and stability of closed orbits in multisector models of optimal economic growth," Journal of Economic Theory, vol. 21, no. 3, pp. 421-444, 1979.

[16] J. Benhabib and R. H. Day, "Erratic accumulation," Economics Letters, vol. 6, no. 2, pp. 113-117, 1980.

[17] J. Benhabib and R. H. Day, "A characterization of erratic dynamics in, the overlapping generations model," Journal of Economic Dynamics and Control, vol. 4, pp. 37-55, 1982.

[18] J.-M. Grandmont, "On endogenous competitive business cycles," Econometrica, vol. 53, no. 5, pp. 995-1045, 1985.

[19] W. A. Brock, "Distinguishing random and deterministic systems: abridged version," Journal of Economic Theory, vol. 40, no. 1, pp. 168-195, 1986.

[20] D. S. Huang and H. Q. Li, Theory and Method of the Nonlinear Economics, Sichuan University Press Co., Ltd., Chengdu, China, 1993.

[21] J.-H. Ma and Y.-S. Chen, "Study for the bifurcation topological structure and the global complicated character of a kind of nonlinear finance system (i)," Applied Mathematics and Mechanics, vol. 22, no. 11, pp. 1240-1251, 2001.

[22] J.-H. Ma and Y.-S. Chen, "Study for the bifurcation topological structure and the global complicated character of a kind of nonlinear finance system (ii)," Applied Mathematics and Mechanics, vol. 22, no. 12, pp. 1375-1382, 2001.

[23] F. Xu, Y. Lai, and X.-B. Shu, "Chaos in integer order and fractional order financial systems and their synchronization," Chaos, Solitons \& Fractals, vol. 117, pp. 125-136, 2018.

[24] F. Zhang, G. Yang, Y. Zhang, X. Liao, and G. Zhang, "Qualitative study of a 4D chaos financial system," Complexity, vol. 2018, Article ID 3789873, 5 pages, 2018.

[25] D. Fu, H.-T. Zhang, A. Dutta, and G. Chen, "A cooperative distributed model predictive control approach to supply chain management," IEEE Transactions on Systems, Man, and Cybernetics: Systems, 2019.

[26] L. Yan, F. Xu, J. Liu, K. L. Teo, and M. Lai, "Stability strategies of demand-driven supply networks with transportation delay," Applied Mathematical Modelling, vol. 76, pp. 109-121, 2019.

[27] H. Xia, "Improve the resilience of multilayer supply chain networks,", Complexity, vol. 2020, Article ID 6596483, 9 pages, 2020. 